\title{
La sécrétion nectarifère des fleurs de cybrides mâles-stériles de colza d'hiver (Brassica napus L)
}

\author{
J Mesquida 1, MH Pham-Délègue 2, R Marilleau 2, M Le Métayer 2, M Renard 3
}

\begin{abstract}
${ }^{1}$ INRA, centre de recherches de Rennes, laboratoire de zoologie, domaine de la Motte au Vicomte, BP 29, 35650 Le Rheu; 2 INRA-CNRS (URA 1190), laboratoire de neurobiologie comparée des invertébrés, La Guyonnerie, 91440 Bures-sur-Yvette; ${ }_{3}$ INRA, centre de recherches de Rennes, station d'amélioration des plantes, domaine de la Motte au Vicomte,
\end{abstract} BP 29, 35650 Le Rheu, France

(Reçu le 10 mai 1990; accepté le 21 janvier 1991)

\begin{abstract}
Résumé - Les sécrétions nectarifères des fleurs de 5 cybrides $(27,58,77,85$ et 118$)$ mâles-stériles de colza d'hiver (Brassica napus $\mathrm{L}$ ) et de la variété Darmor mâle-fertile, ont été comparées après une analyse de leur composition glucidique par chromatographie liquide à haute pression. Par rapport à Darmor, les cybrides ont sécrété moins de nectar ( $50 \%$ de moins pour 27,58 et 85 , et $90 \%$ de moins pour 77 et 118 ), mais les concentrations de leurs constituants glucidiques n'ont pas varié de façon significative. Selon les lieux, les dates et l'heure du prélèvement, seules les quantités de nectar recueillies et les concentrations en matière sèche ont varié. Les concentrations en glucose et fructose sont restées voisines de $50 \%$ sans variation significative; le saccharose a été peu ou pas représenté. Ainsi, bien que d'importantes variations de quantités sécrétées puissent survenir, la composition glucidique des nectars des cybrides apparaît stable et satisfaisante dans la perspective d'une pollinisation entomophile.
\end{abstract}

colza / Brassica napus / cybride / stérilité mâle cytoplasmique / nectar / sucre

Summary - The floral nectar secretion in male-sterile cybrid of winter rapeseed (Brassica napus $\mathrm{L}$ ). Floral nectar secretions of 5 male sterile cybrids of winter rapeseed $(25,58,77,85,118)$ and the Darmor variety were compared after pipette collection and analysis of their sugar composition using high pressure liquid chromatography. The influence of various parameters was studied. Regarding the effect of time of collection (fig 1, table 1): the nectar secretions at 8 am were more abundant (mean of $60 \%$ ) and less concentrated $(\approx 1 / 3)$ than those at $2 \mathrm{pm}$; the relative concentrations of each sugar (glucose, fructose) were similar at $8 \mathrm{am}$ and $2 \mathrm{pm}$ and close to 50\%. Compared to Darmor, cybrids produced less nectar (50\% less for 27, 58, 85 and $90 \%$ less for 77 and 118). Regarding the effect of the day of collection (fig 2, table II): on average, the secretions were more abundant on May 3rd than on April 15th (23\% more). Independently of the day, Darmor secreted more than the cybrids (65-95\% more). The dry matter (19.3-22.4 $\mathrm{mg} / 100 \mu \mathrm{l})$ and the mean concentrations of each sugar $(\approx 50 \%)$ were similar every day. Regarding the combined effect of the day and location (fig 3, tables III, IV): for every day and location, secretions were similar within cybrids and lower than that in Darmor (54-58\% less). Amounts secreted (2-4 $\mu$ l on average) and total sugar amounts $\approx 15-40$ $\mathrm{mg} / 100 \mu \mathrm{l}$ ) differed according to day and location, whereas the relative concentrations of each sugar remained constant. Thus, although large fluctuations might occur in the amounts of nectar, the glucidic composition of the nectars appeared to remain constant in each cybrid and favorable for entomophilous pollination.

rapeseed / Brassica napus / cybrid / cytoplasmic male sterility / nectar / sugar 


\section{INTRODUCTION}

Pour la création de colzas (Brassica napus L) hybrides, l'utilisation de la stérilité mâle s'est révélée nécessaire en raison de l'autogamie prépondérante de cette plante. Des cybrides mâlesstériles (stérilité mâle cytoplasmique ou SMC du type radis (Ogura, 1968) améliorée par fusion de protoplastes, Pelletier et al, 1983), ont été obtenus. Ceux-ci doivent être pollinisés par des plantes mâles fertiles qui fournissent le pollen par l'intermédiaire d'insectes vecteurs de pollen, principalement l'abeille domestique (Apis mellife$r a \mathrm{~L}$ ). Dans un tel système, une bonne pollinisation dépend essentiellement des facteurs d'attractivité et en particulier des sécrétions nectarifères des 2 génotypes en présence. Leur production respective de nectar doit être suffisante quantitativement et qualitativement pour assurer un butinage alterné sur les 2 génotypes. En effet, les abeilles délaissent les génotypes pauvres en nectar au profit des plus nectarifères, ainsi que cela a été observé chez le colza mâlestérile d'origine radis, fortement déficient en nec$\operatorname{tar}$ (Mesquida et Renard, 1978).

De nombreux auteurs (Beutler, 1953; Heinrich, 1975; Baker et Baker, 1975; Harborne, 1982; Masson, 1983; Mesquida et al, 1988 a) ont souligné l'importance des critères qualitatifs et quantitatifs caractérisant les sécrétions nectarifères sur le comportement de butinage des insectes pollinisateurs. Chez le tournesol, par exemple, des études associant des approches biochimiques et comportementales conduites en conditions naturelles et au laboratoire, ont permis de démontrer l'importance de la composition glucidique, en particulier de la présence de saccharose, sur les préférences intergénotypes de l'abeille (Pham-Délègue et al, 1985; Fonta et al, 1985). Ainsi, afin de prendre en compte de tels critères d'attractivité chez différents cybrides mâles-stériles de colza en production de semences hybrides $F_{1}$ et leur utilisation dans un système de variétés «mixtes» (Renard et al, 1988), nous avons entrepris l'étude de l'influence du génotype, du lieu, de la date et de l'heure de prélèvement, sur la quantité et la composition glucidique des sécrétions nectarifères.

Dans un travail antérieur (Mesquida et al, 1988 b), l'analyse de la composition glucidique de nectar de colza a été réalisée en utilisant la technique de chromatographie en phase gazeuse, qui permet un dosage quantitatif précis et des identifications fiables. Toutefois, cette technique est lourde à mettre en œuvre en raison de la nécessité de transformer les sucres en dérivés silylés ou acétylés (Brohst et Lott, 1966;
Bosi, 1973), et les temps d'analyse sont longs. C'est pourquoi nous avons opté pour une technique d'analyse par chromatographie liquide à haute pression (CLHP), développée dans le domaine alimentaire notamment (Thean et Fundersburk, 1977; Black et Bagley, 1978; Quemener et Mercier, 1980), et appliquée efficacement à l'analyse des sucres de nectars floraux (Erickson et al, 1979; Severson et Erickson, 1983).

\section{MATÉRIEL ET MÉTHODES}

Les essais expérimentaux et les prélèvements ont été effectués en 1988 sur le domaine INRA du Rheu (Bretagne). Les analyses chimiques ont été réalisées au laboratoire de neurobiologie comparée des invertébrés INRA-CNRS de Bures-sur-Yvette.

\section{Matériel végétal}

Cinq cybrides mâles-stériles de la lignée de colza d'hiver Darmor (ou plantes femelles): $27,58,77,85$ et 118 (SMC d'origine radis Ogura, 1968, améliorée par fusion de protoplastes, Pelletier et al, 1983) et la même lignée mâle-fertile Darmor (ou plantes mâle) ont été utilisés. Quatre parcelles renfermant chacune ces 5 cybrides mâles-stériles en mélange avec Darmor, dans des proportions variables ont été constituées. Ces parcelles étaient éloignées les unes des autres de plusieurs centaines de $\mathrm{m}$. Les essais variétés “mixtes» (Renard et al, 1988) ont été effectués dans le but de valoriser la stérilité mâle issue de fusion de protoplastes en absence de restaurateur de la fertilité chez l'hybride.

\section{Prélèvements de nectar}

Les prélèvements de nectar ont été effectués par pipetage sur un échantillon de 5 plantes (correspondant aux répétitions) par génotype, à raison de 2-5 fleurs par plante. Le pipetage a été réalisé à l'aide de micropipettes graduées d'une contenance de $5 \mu$ l (diamètre interne : $1 \mathrm{~mm}$ ), l'estimation des quantités prélevées se faisant par lecture directe de la colonne de liquide. Les résultats ont été exprimés en quantité de nectar par fleur. Après chaque prélèvement, les pipettes ont été systématiquement bouchées aux 2 extrémités et stockées au froid $\left(-20^{\circ} \mathrm{C}\right)$. Préalablement aux prélèvements, les plantes ont été recouvertes d'un sac en papier durant $24 \mathrm{~h}$, afin d'éviter le butinage du nectar par les pollinisateurs.

\section{Facteurs étudiés}

- l'heure du prélèvement ( $8 \mathrm{~h}$, heure solaire, et $14 \mathrm{~h}$, 21 avril); 
- la date du prélèvement (15 avril et 3 mai) d'une même parcelle;

- la combinaison de la date et du lieu sur des prélèvements de nectar effectués entre le 15 avril et le 5 mai (prélèvements successifs sur chaque parcelle les 15 , 21 et 27 avril et le 5 mai).

\section{Analyse des sucres du nectar}

Le système analytique se compose d'un chromatographe liquide à haute pression Waters, équipé d'un module de pompe Waters $6000 \mathrm{~A}$, d'un injecteur U6K Waters à double boucle, d'une colonne radial-Pak (10 $\times 8 \mathrm{~mm}$ ) DI garnie de silice $10 \mu \mathrm{m}$, d'un détecteur réfractométrique ERMA 7510 haute sensibilité et d'un intégrateur enregistreur Delsi Enica 10. Le système comprend en outre un préfiltre mécanique, un module Guard-Pak équipé d'une précolonne de silice, un module Waters RCM 100 à compression radiale, un kit de filtration pour solvants (filtres $0,22 \mu \mathrm{m}$ ) et d'une cuve à ultrasons Bransonic 32.

Les solvants utilisés sont l'acétonitrile pour CLHP (Carlo-Erba), de l'eau ultrapure Milli Q (Millipore), de la tétraéthylènepentamine à $20 \%$ (Merck) et du méthanol (Prolabo).

Une solution mère est préparée à partir d'un mélange eau-acétonitrile $(50 / 50)$ additionné d'un étalon interne, le rhamnose $(2 \mathrm{mg} / \mathrm{ml})$. Les sucres de référence (glucose-fructose-saccharose, Merck, Sigma) sont incorporés à la concentration de $1 \mathrm{mg} / \mathrm{ml}$ de la solution mère, pour former une solution témoin. Les échantillons sont pesés et dilués aussitôt dans $2 \mathrm{ml}$ de solution mère, et filtrés à l'aide de Swinnex sur filtre hydrophile $0,22 \mu \mathrm{m}$.

Des volumes constants $(20 \mu \mathrm{l})$ et répétés du mélange de référence, puis un volume égal de la solution d'échantillon à doser sont injectés. La concentration de chaque sucre en poids réel par rapport au poids de nectar analysé $(\mathrm{mg} / 100 \mathrm{mg}$ ) est obtenue par une méthode de calcul décrite par ailleurs (Black et Bagley, 1978; Vear et al, 1990). En référence au travail de Bolten et al (1979), qui préconisent l'adoption d'une expression standard des teneurs en sucres des nectars, les résultats ont été exprimés en concentration de sucres dans le nectar $(\mathrm{mg} / 100 \mu \mathrm{l})$ après conversion d'après les abaques présentés par Weast et al (1988) (tableaux 21, 22, 88). Les concentrations des sucres constitutifs de la matière sèche sont exprimées en proportions relatives ( $\%$ des sucres totaux).

\section{Analyse des données}

Les analyses de la variance ont été réalisées sur les valeurs moyennes par fleur et pour 5 plantes (correspondant aux répétitions), avec le logiciel statistique STAT-ITCF (Göuet et al, 1985), selon un modèle à effets fixés en prenant en compte 3 facteurs : génotype (correspondant aux génotypes 27, 58, 85, Darmor), date ou h de prélèvement du nectar et bloc. Dans le cas du rejet de l'hypothèse nulle, l'analyse de la variance a été suivie d'un test de classification de moyennes à la probabilité $P=0,05$ de Newman et Keuls avec le logiciel STAT-ITCF. Les pourcentages ont été analysés après transformation angulaire (Arcsinus VP).

Les faibles quantités de nectar recueillies sur les cybrides 77 et 118 , présentes souvent sous forme de traces non mesurables, sont exclues des analyses.

\section{RÉSULTATS}

\section{Effet de l'heure du prélèvement}

\section{Quantités de nectar}

Bien que l'effet combiné des facteurs génotype et $\mathrm{h}$ de prélèvement ne soit pas significatif (tableau I, NF), ces effets apparaissent hautement significatifs lorsqu'ils sont considérés séparément : les productions de nectar de $8 \mathrm{~h}$ sont plus abondantes que celles de $14 \mathrm{~h}(60 \%$ en moyenne, fig $1 \mathrm{~A}, \mathrm{NF}$ ); parmi les génotypes, les sécrétions de Darmor sont plus abondantes que celles des cybrides (94-121\% en moyenne, fig 1 $\mathrm{B}, \mathrm{NF}$ ). Les sécrétions nectarifères de 118 et de 77 ayant été nulles ou faibles (moins de $1 \%$ de Darmor pour 77 et de 0,5 à $10 \%$ de Darmor pour $118)$, ces 2 cybrides ne seront pas considérés dans la suite des analyses.

\section{Concentrations en sucres totaux}

Les faibles sécrétions de nectar recueillies à $14 \mathrm{~h}$ apparaissent plus concentrées en sucres totaux que celles plus abondantes de $8 \mathrm{~h}(184 \%$ en moyenne, fig $1 \mathrm{~A}, \mathrm{MS}$ ). L'effet h est lié à une augmentation significative des concentrations en sucres des génotypes 58 et Darmor (fig $1 \mathrm{~A}$, MS) $(205 \%$ et $270 \%$ d'augmentation, respectivement, tableau I, MS).

\section{Concentrations relatives en sucres}

Les concentrations moyennes de chacun des sucres apparaissent très voisines entre $8 \mathrm{~h}$ et $14 \mathrm{~h}$, valeurs proches de $50 \%$ (fig $1 \mathrm{~A}$, FR et $\mathrm{GL}$ ). Pour le fructose, il n'apparaît pas d'effet génotype significatif (fig $1 \mathrm{~B}, \mathrm{FR}$ ), bien qu'il existe un effet significatif de l'interaction heure $x$ génotype, lié à une différence de concentration des génotypes 27 et 85 à $14 \mathrm{~h}$ (tableau I, FR). Pour le glucose, une interaction heure-génotype significative apparaît (tableau I, GL), avec une concentration en moyenne plus faible chez Dar- 
Tableau I. Production de nectar par fleur, teneur en matière sèche, concentrations relatives en fructose et glucose du nectar des génotypes de colza, selon l'heure de prélèvement (21 avril). Moyennes par fleur et pour 5 plantes (correspondant aux répétitions), suivies de l'erreur type (chiffres entre parenthèses). NF = quantités de nectar par fleur (en $\mu \mathrm{l}$ ); MS = teneur en matière sèche du nectar (en $\mathrm{mg} / 100 \mu \mathrm{l}) ; \mathrm{FR}=$ concentrations relatives en fructose (en $\%$ de la matière sèche); $\mathrm{GL}=$ concentrations relatives en glucose (en $\%$ de la matière sèche); DIF = différences (test $F$ à 3 et $28 \mathrm{ddl}$ ): NS : valeur non significative; $\mathrm{S}^{\star}$ valeur significative à $P<0,05$; $\mathrm{S}^{\star \star}$ valeur significative à $P<0,01$; $\mathrm{S}^{\star \star \star}$ valeur significative à $P<0,001 ; \mathrm{CV}=$ coefficient de variation. Les moyennes suivies d'une même lettre ne sont pas significativement différentes à la probabilité $P<0,05$ (Test de Newman-Keuls).

\begin{tabular}{|c|c|c|c|c|c|}
\hline & Heures & & Génotypes & & \\
\hline & & 27 & 58 & 85 & Darmor \\
\hline$N F$ & $\begin{array}{l}08 \text { h00 } \\
14 \text { h00 } \\
\text { Dif }\end{array}$ & $\begin{array}{l}1,7 \\
(0,6) \\
1,5 \\
(1,2)\end{array}$ & $\begin{array}{l}1,9 \\
(0,5) \\
0,9 \\
(0,7) \\
\text { NS (CV 47\%) }\end{array}$ & $\begin{array}{l}1,8 \\
(0,4) \\
1,4 \\
(0,7)\end{array}$ & $\begin{array}{l}4,1 \\
(1,3) \\
2,1 \\
(2,1)\end{array}$ \\
\hline MS & $\begin{array}{l}08 \text { h } 00 \\
14 \text { h } 00 \\
\text { Dif }\end{array}$ & $\begin{array}{l}11,9 \mathrm{c} \\
(1,8) \\
29,1 \mathrm{~b} \\
(7,3)\end{array}$ & $\begin{array}{l}13,1^{c} \\
(1,7) \\
48,6^{a} \\
(6,5) \\
S^{\star}(C \vee 36,5 \%)\end{array}$ & $\begin{array}{l}12,4^{c} \\
(1,3) \\
25,3^{b} \mathrm{c} \\
(5,6)\end{array}$ & $\begin{array}{l}18 b^{c} \\
(1,2) \\
54,9 a \\
(6,8)\end{array}$ \\
\hline$F R$ & $\begin{array}{l}08 \text { h } 00 \\
14 \text { h } 00 \\
\text { Dif }\end{array}$ & $\begin{array}{l}49,8^{a b} \\
(1,5) \\
46,5^{b} \\
(1,7)\end{array}$ & $\begin{array}{l}47,1^{\mathrm{ab}} \\
(1,9) \\
47,3^{\mathrm{ab}} \\
(0,5) \\
\mathrm{S}^{\star \star \star}(\mathrm{CV} 4 \%)\end{array}$ & $\begin{array}{l}47,3^{a b} \\
(2,2) \\
50,7^{a} \\
(1,2)\end{array}$ & $\begin{array}{l}48,2^{a b} \\
(3,3) \\
48,9^{a b} \\
(0,9)\end{array}$ \\
\hline$G L$ & $\begin{array}{l}08 \text { h } 00 \\
14 \text { h } 00 \\
\text { Dif }\end{array}$ & $\begin{array}{l}49,1^{b c} \\
(1,5) \\
53,5^{a} \\
(1,7)\end{array}$ & $\begin{array}{l}52,9 \mathrm{a} \\
(1,9) \\
51,1 \mathrm{ab} \\
(1,6) \\
\mathrm{S}^{\star \star \star}(\mathrm{CV} 3 \%)\end{array}$ & $\begin{array}{l}52,7^{\mathrm{a}} \\
(2,4) \\
49,3^{\mathrm{bc}} \\
(1,2)\end{array}$ & $\begin{array}{l}47,5^{c} \\
(2,6) \\
50,7 a^{b} \\
(0,8)\end{array}$ \\
\hline
\end{tabular}
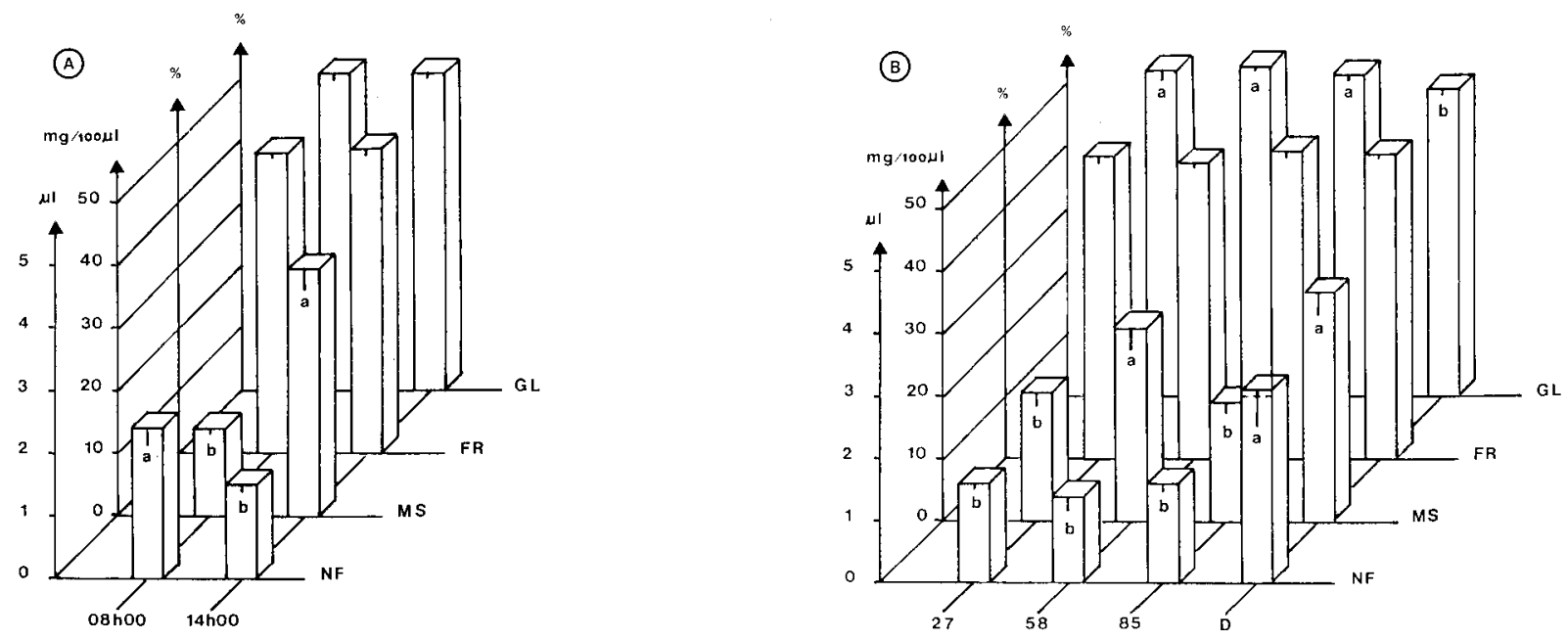

Fig 1. Valeurs moyennes des productions de nectar par fleur, des teneurs en matière sèche, et des concentrations relatives en fructose et glucose (A) en fonction de l'heure (B) en fonction du génotype. Même légende que tableau I. (A) : test $F$ à 1 et 28 ddl; NF:




mor (fig 1, B, GL), liée à une proportion relativement plus élevée en saccharose. En effet, les quantités de saccharose de Darmor sont en moyenne de $4,3 \%$ à $8 \mathrm{~h}$, et $0,4 \%$ à $14 \mathrm{~h}$, alors que celles des cybrides sont de l'ordre de $1,1 \%$ à $8 \mathrm{~h}$ pour 27 , et de $1,6 \%$ pour 58 à $14 \mathrm{~h}$.

\section{Effet de la date de prélèvement}

\section{Quantités de nectar}

La figure $2 \mathrm{~A}, \mathrm{~B}(\mathrm{NF})$ met en évidence un effet date et un effet génotype hautement significatifs.

Tableau II. Production de nectar par fleur, teneur en matière sèche, concentrations relatives en fructose et glucose du nectar des génotypes de colza, selon la date de prélèvement, sur une même parcelle. Même légende que tableau I.

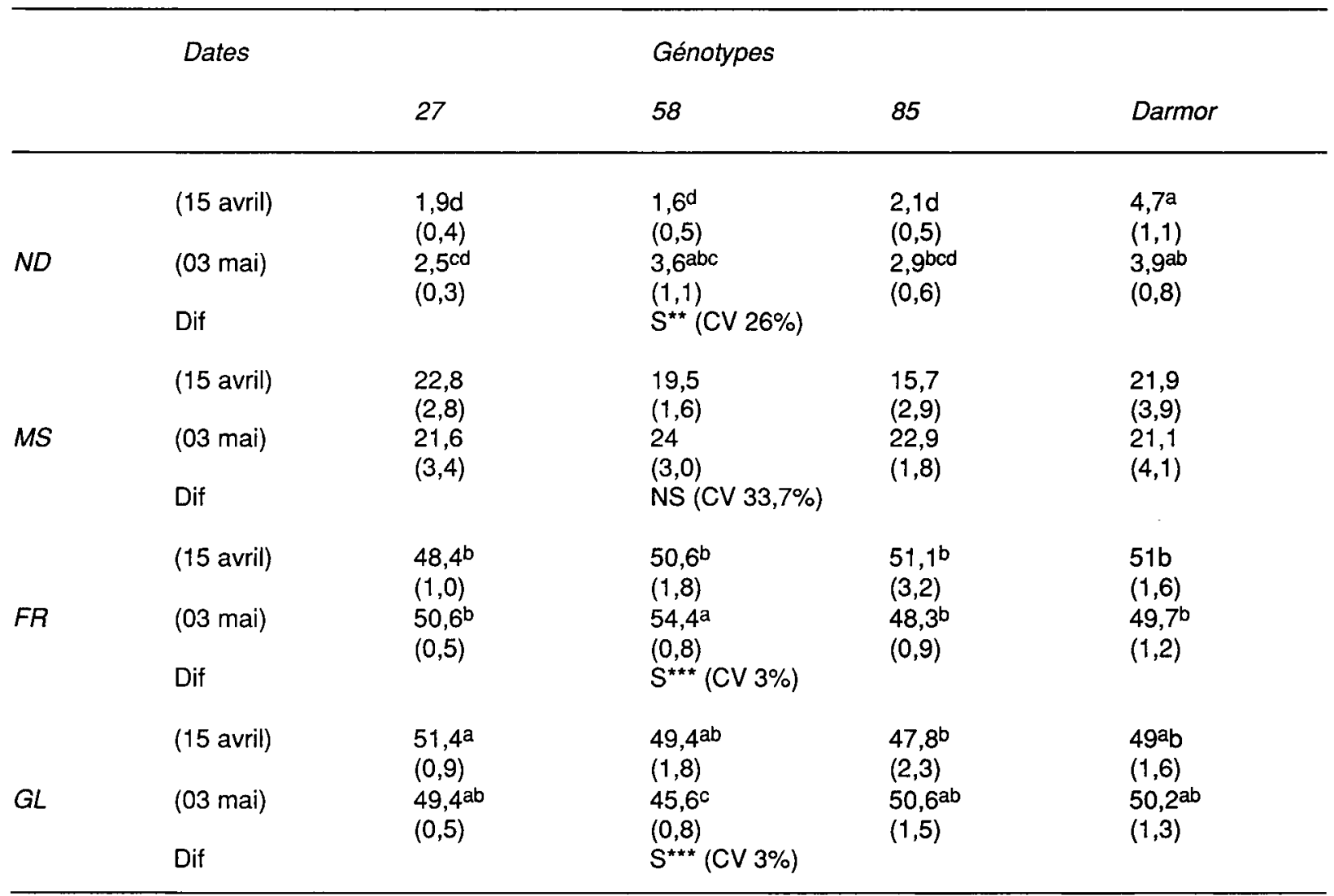
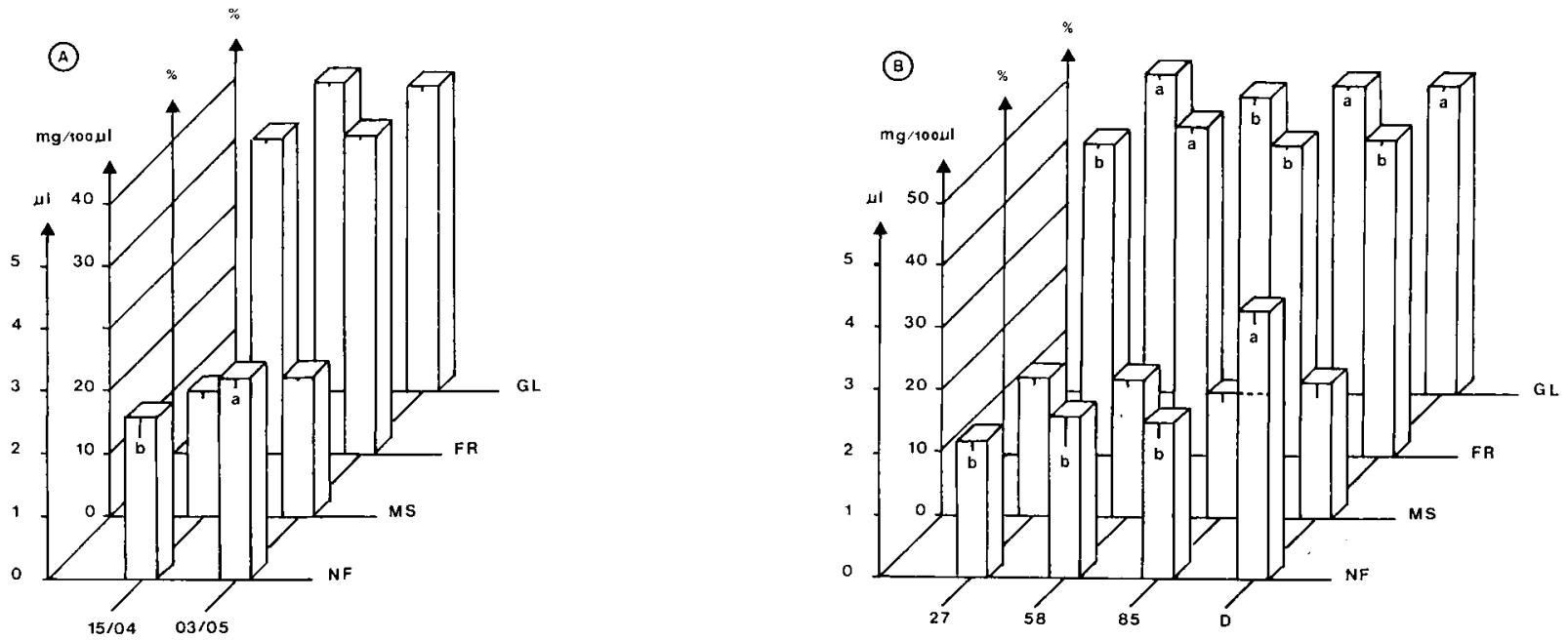

Fig 2. Valeurs moyennes des productions de nectar par fleur, des teneurs en matière sèche, et des concentrations relatives en fructose et glucose, (A) en fonction de la date, (B) en fonction du génotype. Même légende que tableau I. (A): test $F$ à 1 et 28 ddl; NF: S**; MS: NS; FR: NS; GL: NS; (B): test $F$ à 3 et 28 ddl; NF: S***; MS: NS; FR: S***; GL: S**. 
L'interaction des 2 effets est très significative (tableau II, NF). En moyenne les quantités de nectar prélevées le 3 mai apparaissent plus élevées que celles du 15 avril ( $23 \%$ en plus). L'effet date est particulièrement sensible pour le génotype 58 (augmentation de 125\%). Les conditions météorologiques enregistrées à ces 2 dates ont été cependant assez voisines (températures moyennes de $12,9^{\circ} \mathrm{C}$ le 15 avril avec minimum de $10,7{ }^{\circ} \mathrm{C}$ et maximum de $15,2{ }^{\circ} \mathrm{C}$, et de $12,8^{\circ} \mathrm{C}$ le 3 mai avec un minimum de $10,2^{\circ} \mathrm{C}$ et un maximum de $15,4^{\circ} \mathrm{C}$ ). L'effet lignée est essentiellement dû aux faibles sécrétions nectarifères des cybrides par rapport à celles de Darmor (fig 2 B, NF) (de $65 \%$ à $95 \%$ en plus chez Darmor).

\section{Concentrations en sucres totaux}

Les concentrations en sucres totaux pour chacune des lignées et pour chacune des dates sont équivalentes et comprises entre 19,3 et $22,4 \mathrm{mg} / 100 \mu \mathrm{l}$ (tableau II, MS et fig $2 \mathrm{~A}, \mathrm{~B}, \mathrm{MS}$ ).

\section{Concentrations relatives en sucres}

Les concentrations moyennes de chacun des sucres, et pour chacune des dates de prélèvement, sont voisines et proches de $50 \%$ (tableau II, FR et GL; fig 2 A, FR, GL). On met cependant en évidence des différences significatives entre génotypes le 3 mai, 58 ayant des teneurs plus élevées en fructose, et inversement moins élevées en glucose (fig $2 \mathrm{~B}, \mathrm{FR}$ et $\mathrm{GL}$ ).

Les traces de saccharose identifiées sont de l'ordre de 0,2 et $0,1 \%$ pour 27 et 85 , pour les prélèvements du 15 avril, et de 1,1 et $0,1 \%$ pour 85 et Darmor, pour ceux du 3 mai.

\section{Effet combiné de la date et du lieu}

\section{Quantités de nectar}

Les valeurs moyennes des productions de nectar des différents génotypes cultivés dans différentes parcelles font apparaître un effet génotype et un effet lieu-date hautement significatifs (fig 3 A et $B, N F$ ).

Pour chaque lieu-date (tableau III, NF), et en moyenne (fig $3 \mathrm{~B}, \mathrm{NF}$ ), les cybrides ont sécrété des quantités de nectar équivalentes entre elles et significativement plus faibles que celles de Darmor ( $54 \%-58 \%$ de moins en moyenne).

C'est dans la parcelle 3 (27 avril) que les valeurs sont minimales, et dans la parcelle 4 ( 5 mai) qu'elles sont maximales; celles des parcelles 1 et 2 apparaissent intermédiaires et peu différentes entre elles (tableau III, NF).

Tableau ill. Production de nectar par fleur, teneur en matière sèche, selon les lieux et dates de prélèvement. Même légende que tableau I. Test $F$ à 9 et 60 ddl.

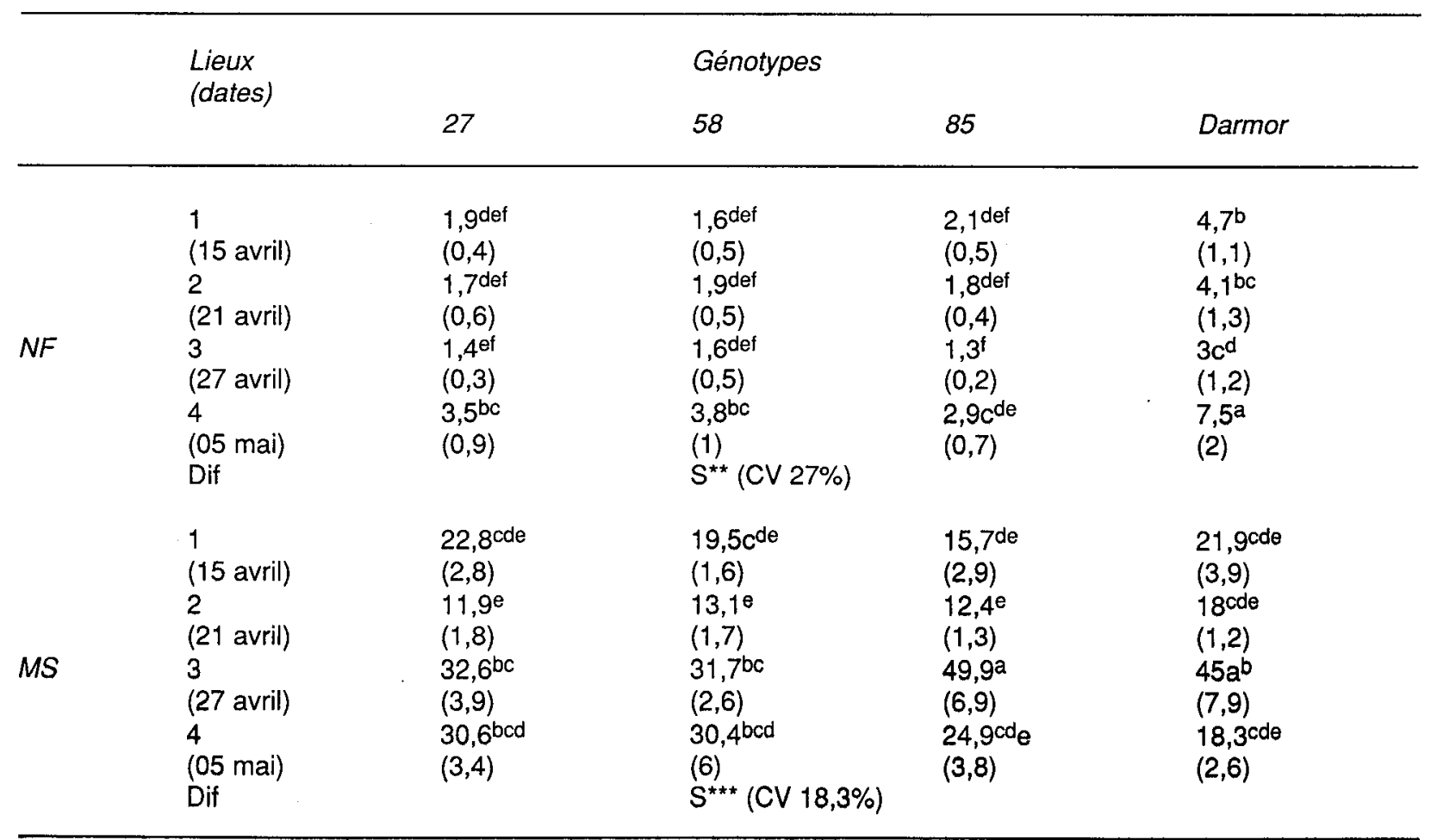



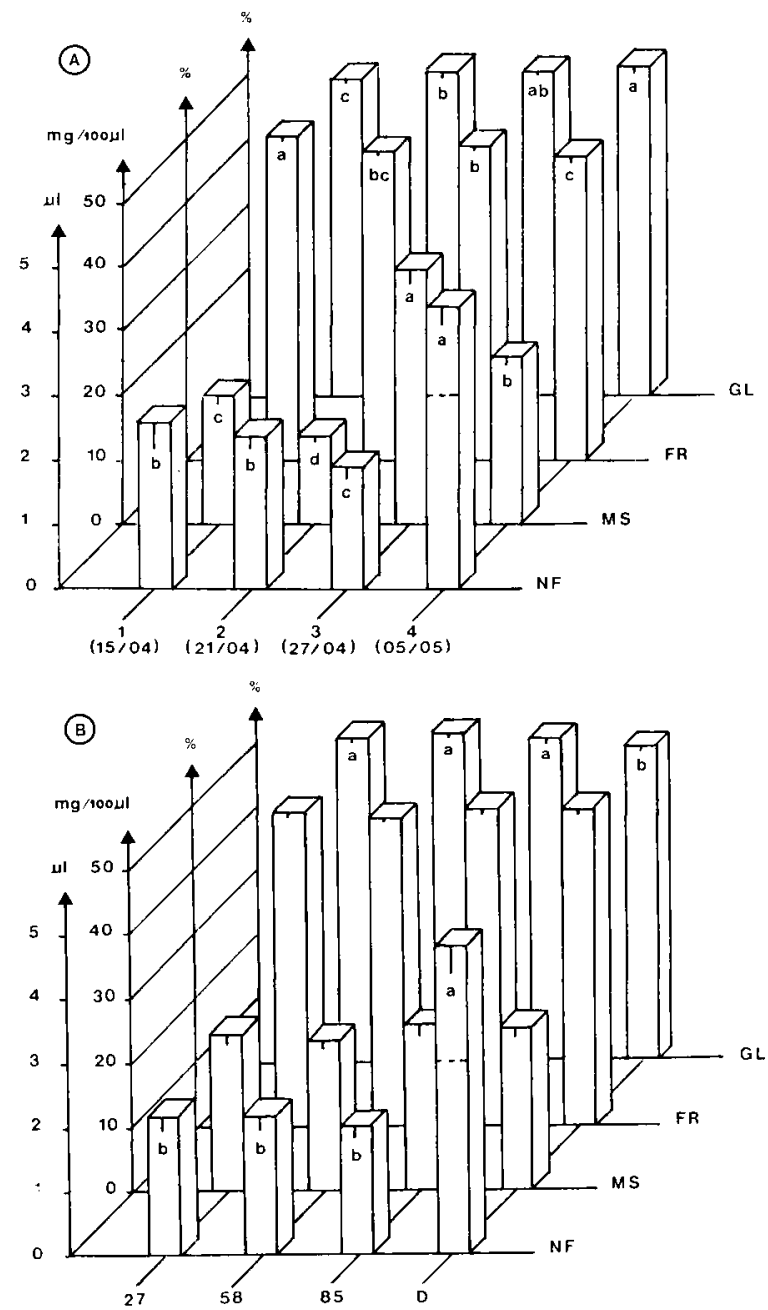

Fig 3. Valeurs moyennes des productions de nectar par fleur, des teneurs en matière sèche, et des concentrations relatives en fructose et glucose, (A) en fonction des lieux et dates, (B) en fonction des génotypes. Même légende que tableau I. (A) test $F$ à 3 et 60 ddl; NF: $S^{* * *} ; M S: S^{* \star *} ;$ FR: $S^{* * *} ;$ GL: $S^{* * *}$; (B): test $F$ à 3 et 60 ddl; NF: $S^{* * * ;}$ MS: NS; FR: NS; GL: $S^{* * *}$.

\section{Composition glucidique}

L'analyse chromatographique a révélé la présence de 2 sucres : fructose et glucose. Du saccharose a été identifié dans quelques échantillons en très faibles quantités ou sous forme de traces.

\section{Concentrations en sucres totaux}

L'interaction des effets génotypes de lieu-date apparaît hautement significative (tableau III, MS). Ceci est dû à un effet lieu-date hautement significatif, les valeurs les plus élevées correspondant à la parcelle 3 (27 avril) et les plus faibles à la parcelle 2 (21 avril) (fig 3 A, MS). L'effet lignée n'apparaît pas significatif en moyenne ( $f$ B, MS).

\section{Concentrations relatives en sucres}

Les concentrations relatives en chacun des sucres dans la matière sèche du nectar (tableau II) sont globalement peu différentes de $50 \%$ quels que soient les génotypes et les lieux-dates (tableau IV); le glucose de chaque génotype est sensiblement plus abondant (de l'ordre de 5$17 \%$ de plus que le fructose) dans 11 cas sur 16.

L'analyse statistique fait apparaître un effet génotype pour le glucose seulement, et un effet lieu-date pour le fructose et le glucose (fig $3 \mathrm{~A}$ et $B, F R$ et GL). Parmi les génotypes, Darmor présente la teneur moyenne en glucose la plus faible (fig $3 \mathrm{~B}, \mathrm{GL}$ ). Parmi les lieux-dates, la parcelle 1 (15 avril) présente les teneurs les plus élevées en fructose et les plus faibles en glucose, et inversement pour la parcelle 4 (fig $3 \mathrm{~A}$, FR et $G L)$.

Les petites quantités de saccharose identifiées sont de l'ordre de $0,2-1,1 \%$ pour 27 et 85 , parcelle 1 ( 15 avril); de 1,1 à $4,3 \%$ pour 27 et Darmor, parcelle 2 ( 21 avril); de $0,6 \%$ pour 27 , parcelle 3 (27 avril), et de 0,3 à $2,2 \%$ pour 27 , $58,85,118$ et Darmor, parcelle 4 (5 mai).

\section{DISCUSSION ET CONCLUSION}

La composition en sucres des nectars, peut être analysée par différentes techniques telles que la chromatographie sur papier (Wykes, 1952), la chromatographie en phase gazeuse (Bosi, 1973; Mesquida et al, 1988 b), et la chromatographie liquide à haute pression (Erickson et al, 1979; Severson et Erickson, 1983). Dans ce travail, les échantillons de colza ont été analysés par CLHP; les résultats obtenus par cette méthode s'avèrent comparables à ceux obtenus précédemment par chromatographie en phase gazeuse pour la même espèce (Mesquida et al, 1988 b). Ainsi, la présence de 2 sucres principaux, glucose et fructose, a été mise en évidence, des traces de saccharose ont de même été identifiées ici par CLHP, cette composition étant conforme à celle rapportée pour Brassica napus par Wykes (1952) et Percival (1961). En comparaison avec la chromatographie en phase gazeuse, la méthode d'analyse par CLHP permet de traiter rapidement un grand nombre d'échantillons, à partir de faibles quantités (de l'ordre de $1 \mu \mathrm{l}$ ), sans préparation préalable. La CLHP apparaît donc particulièrement adaptée à la caractérisation de nectars floraux, ainsi que l'avaient préconisé Severson et Erickson (1983). 
Tableau IV. Concentrations relatives en fructose et glucose, selon les lieux et dates de prélèvement. Même légende que tableau I. Test $F$ à 9 et $60 \mathrm{ddl}$.

\begin{tabular}{|c|c|c|c|c|c|}
\hline & \multirow{2}{*}{$\begin{array}{l}\text { Lieux } \\
\text { (dates) }\end{array}$} & \multicolumn{3}{|c|}{ Génotypes } & \multirow[b]{2}{*}{ Darmor } \\
\hline & & 27 & 58 & 85 & \\
\hline$F R$ & $\begin{array}{l}1 \\
\text { (15 avril) } \\
2 \\
(21 \text { avril) } \\
3 \\
\text { (27 avril) } \\
4 \\
\text { (05 mai) } \\
\text { Dif }\end{array}$ & $\begin{array}{l}48,4 \\
(1) \\
49,8 \\
(1,5) \\
48,4 \\
(0,7) \\
48 \\
(0,7)\end{array}$ & $\begin{array}{l}50,6 \\
(1,9) \\
47,1 \\
(1,9) \\
48,9 \\
(0,7) \\
45,9 \\
(1,9) \\
\text { NS (CV 4\%) }\end{array}$ & $\begin{array}{l}51,1 \\
(3,2) \\
47,3 \\
(2,1) \\
49 \\
(0,6) \\
47,1 \\
(1,1)\end{array}$ & $\begin{array}{l}51 \\
(1,6) \\
48,2 \\
(3,3) \\
49,5 \\
(0,6) \\
48,4 \\
(5,6)\end{array}$ \\
\hline$G L$ & $\begin{array}{l}1 \\
\text { (15 avril) } \\
2 \\
(21 \text { avril) } \\
3 \\
\text { (27 avril) } \\
4 \\
\text { (05 mai) } \\
\text { Dif }\end{array}$ & $\begin{array}{l}51^{\mathrm{ab}} \\
(0,9) \\
49,1^{\mathrm{bc}} \\
(1,5) \\
51^{\mathrm{ab}} \\
(0,7) \\
51,4^{\mathrm{ab}} \\
(1,2)\end{array}$ & $\begin{array}{l}49,4^{\mathrm{abc}} \\
(1,8) \\
52,9^{\mathrm{a}} \\
(1,9) \\
51,1^{\mathrm{ab}} \\
(0,7) \\
52,6^{\mathrm{a}} \\
(2) \\
\left.\mathrm{S}^{\star \star \star} \text { (CV } 3 \%\right)\end{array}$ & $\begin{array}{l}47,8^{c} \\
(2,3) \\
52,7^{a} \\
(2,4) \\
51 a b \\
(0,6) \\
52,6^{a} \\
(1)\end{array}$ & $\begin{array}{l}49^{\mathrm{bc}} \\
(1,6) \\
47,5^{\mathrm{c}} \\
(2,6) \\
50,5^{\mathrm{abc}} \\
(0,6) \\
51,1^{\mathrm{ab}} \\
(6)\end{array}$ \\
\hline
\end{tabular}

Les résultats obtenus montrent que les sécrétions nectarifères des lignées de colza étudiées peuvent présenter des variations importantes quantitativement et qualitativement, selon les génotypes mais aussi selon les lieux, le moment de la journée et la date de prélèvement.

\section{Selon les génotypes}

Les cybrides ont toujours sécrété des quantités de nectar inférieures à celles du témoin Darmor. Mais certains d'entre eux $(27,58$ et 85$)$ se sont montrés plus nectarifères que d'autres (77 et 118).

Ces différences entre cybrides s'expliquent par le fait qu'ils ne sont pas génotypiquement identiques. A la suite d'une étude comparative de I'ADN mitochondrial, Vedel et al (1987) avaient en effet conclu que les cybrides 77 et 118 se rapprochaient du cytoplasme du type Ogura initial, tandis que 27,58 et 85 se rapprochaient davantage du type colza. Or, nous avons montré (Mesquida et Renard, 1978) que ce cytoplasme Ogura était précisément déficitaire en nectar et par conséquent, difficilement utilisable en production de semences hybrides $F_{1}$. Par contre, les sécrétions nectarifères des cybrides
27 et 58 du type colza ont été estimées suffisamment abondantes pour assurer une répartition homogène des insectes pollinisateurs dans les dispositifs de production de semences hybrides en bandes alternées (Mesquida et al, 1988 a).

Mais si les cybrides ont la particularité de sécréter moins de nectar que le parent mâle-fertile, il est cependant à noter qu'ils se caractérisent par une certaine stabilité nectarifère. En conditions identiques, les quantités de nectar produites sont toujours équivalentes entre cybrides du même type et restent proportionnellement constantes par rapport à celles de leur parent fertile : de l'ordre de $50 \%$ pour les plus nectarifères $(27,58$ et 85$)$, et de moins de $10 \%$ pour ceux qui en produisent moins (77 et 118). Cette stabilité a d'ailleurs été confirmée au cours de diverses mesures effectuées aussi bien sur des génotypes d'hiver (Mesquida, Roger, non publié), que sur ceux du printemps (Bensaoud, Koubaiti, Le Métayer, communication personnelle).

La concentration en matière sèche du nectar des cybrides est par contre peu différente de celle de Darmor, ainsi que les teneurs de leurs constituants glucidiques, glucose et fructose, dont les proportions sont chacune voisines de $50 \%$. 
Selon Jes lieux d'implantation

On observe d'importantes variations dans les quantités de nectar produites par les lignées de colza, ainsi que dans leur concentration en matière sèche, selon les lieux d'implantation de nos essais.

On sait, à la suite des travaux de nombreux auteurs (eg Mitchener, 1927; Beutler, 1953; Huber, 1956; Nunez, 1973; Robacker et al, 1983), que les conditions pédoclimatiques jouent un rôle important dans la régulation des sécrétions nectarifères des fleurs, et que la température et l'humidité de l'air, ainsi que l'humidité du sol (Huber, 1956) et la durée de l'ensoleillement (Shuel, 1955), sont prépondérantes.

\section{Selon le moment de prélèvement}

Huber (1956), Meyerhoff (1958) et Radchenko (1964) rapportent que les sécrétions du colza, très influencées par la température, sont élevées le matin et tard le soir, et faibles à midi; Oertel (1946) et Radchenko (1964) considèrent que le taux de matière sèche du nectar augmente dans la journée avec la température. Si l'on examine les prélèvements d'une même journée et d'un même lieu, il apparaît clairement que les quantités élevées de nectar du matin sont associées aux faibles concentrations en matière sèche, et, inversement, les quantités plus faibles de $14 \mathrm{~h}$ sont associées aux plus fortes concentrations. Ainsi Kleber (1935), Fahn (1949) et Nunez (1973) avaient déjà montré, chez d'autres espèces végétales, que les sécrétions nectarifères, abondantes et faiblement concentrées le matin à température basse et à humidité relative élevée, diminuent progressivement en quantité et deviennent plus concentrées quand la température augmente et que l'humidité de l'air décroît, jusqu'à $14 \mathrm{~h}$, à cause du phénomène d'évaporation. Huber (1956) considère que l'augmentation de la température accroît la production de sucre dans le nectar, jusqu'à atteindre un optimum. Les variations journalières de la quantité de nectar produite et de la teneur en matière sèche ainsi observées dans nos conditions d'essais, semblent résulter d'un phénomène d'évaporation dû à une augmentation de température notamment, ou à d'autres facteurs, tels que l'ensoleillement ou l'humidité de l'air, ainsi que l'ont montré Corbet et al (1979); dans nos conditions expérimentales, les fleurs étant ensachées préalablement aux prélèvements de nectar, il est probable que l'humidité relative à l'intérieur des sacs influence plus directement les variations de concentrations que l'humidité relative extérieure.

\section{Selon les dates de prélèvement}

En un même lieu, et à températures de l'air voisine, les productions de nectar des lignées de colza étudiées dans nos conditions d'expérimentation demeurent constantes, tant en quantité qu'en teneur en matière sèche. On note toutefois une tendance à produire davantage de nectar pendant la période de pleine floraison (le 3 mai) par rapport aux quantités produites en début de floraison (le 15 avril) chez les cybrides (cybride 58 principalement), alors que l'on observe le phénomène inverse chez Darmor. La diminution de sécrétion liée au vieillissement de la plante, signalé par Williams (1980), correspondrait au cas de Darmor mais non à celui des cybrides.

Il apparaît aussi que, si les productions nectarifères des cybrides sont susceptibles de varier largement en fonction des conditions pédoclimatiques, leur profil glucidique demeure constant et caractéristique de l'espèce végétale, avec des taux de fructose et de glucose permettant d'espérer un niveau d'attractivité satisfaisant vis-àvis des insectes pollinisateurs.

Par ailleurs, d'autres constituants biochimiques, dont le rôle dans les préférences florales des pollinisateurs a été montré, tels que les protéines et les acides aminés des nectars (Baker et Baker, 1975, 1983), les ions des nectars (Waller et al, 1972), les lipides et les arômes des pollens (Dobson, 1987; Dobson et al, 1987; Dobson, 1988), et les arômes floraux (PhamDélègue et al, 1986) devront être pris en compte. En effet, ces éléments pourraient permettre de caractériser plus précisément les génotypes et la compréhension de leur rôle dans les choix des insectes pourrait conduire à terme à un criblage ou à une sélection de ces critères en vue d'une optimisation du comportement de butinage.

D'ores et déjà, ce travail nous a permis de mieux appréhender les caractéristiques nectarifères de différents génotypes de colza et d'en envisager les implications possibles sur la pollinisation entomophile des cybrides mâles-stériles utilisables en production de semences hybrides $F_{1}$ et dans un système de variétés «mixtes".

\section{RÉFÉRENCES}

Baker HG, Baker I (1975) Studies of nectar constitution and pollinator-plant coevolution. In: Coevolution of animals and plants. (Gilbert LE, Raven PH, eds) Proc Symp 5 of the 1st Int Congr Syst Evolution Biol Boulder (Colorado), 100-140 
Baker HG, Baker I (1983) A brief historical review of the chemistry of floral nectar. In: The biology of nectaries. (Bentley B, Elias T, eds) Columbia Univ Press, 127-152

Beutler R (1953) Nectar. Bee World 34, 107-162

Black LT, Bagley EB (1978) Determination of oligosaccharides in soybeans by high pressure liquid chromatography using an internal standard. $J A m$ Oil Chem Soc 55, 228-232

Bolten AB, Feinsinger P, Baker HG, Baker I (1979) On the calculation of sugar concentration in flower nectar. Oecologia 41, 301-304

Bosi G (1973) Méthode rapide pour la détermination par chromatographie en phase gazeuse des glucides du nectar : technique de prélèvement du nectar et de préparation de éthers triméthylsilylés en présence d'eau. Apidologie 4, 57-64

Brohst KM, Lott CE (1966) Determination of some components in corn syrup by gas liquid chromatography of the trimethylsilyl derivatives. Cereal Chem 43, 35-43

Corbet SA, Willmer PG, Beatment JWL, Unwin DM, Prys-Jones OE (1979) Post-secretory determinants of sugar concentration in nectar. Plant Cell Environ 2, 293-308

Dobson HEM (1987) Role of flower and pollen aromas. Oecologia 72, 618-623

Dobson HEM (1988) Survey of pollen and pollenkitt lipids-chemical cues to flowers visitors. Ann $J$ Bot 75, 170-182

Dobson HEM, Bergström J, Bergström G, Groth 1 (1987) Pollen and flower volatiles in two Rosa species. Phytochemistry 26, 3171-3173

Erickson EH, Thorp RW, Briggs DL, Estes JR, Daun RJ, Marks M, Schroeder CH (1979) Characterization of floral nectars by high-performance liquid chromatography. J Apic Res 18, 148-152

Fahn A (1949) Studies in the ecology of nectar secretion. Palest J Bot Jerus 4, 207-224

Fonta C, Pham-Délègue MH, Marilleau R, Masson C (1985) Rôle des nectars de tournesol dans le comportement des insectes pollinisateurs et analyse qualitative et quantitative des éléments glucidiques de ces sécrétions. Acta Oecol Oecol Applic 6, 165175

Gouet H, Gouet JP, Phillipeau G, Tranchefort J, Verneau M (1985) Manuel d'utilisation STAT-ITCF. Service des Études Statistiques et Informatiques de l'ITCF, Boigneville II.8.1-II.8.13

Harborne JB (1982) Role of nectar and pollen. In : Introduction to ecological biochemistry. Acad Press, 2nd ed, 32-65

Heinrich B (1975) Energetics of pollination. Ann Rev Ecol Syst 6, 139-170

Huber H (1956) Die Abhangigkeit der Nektarsekretion von temperature, luft -und Bodenfeuchtigkeit. Planta $48,47-98$
Kleber E (1935) Hat das Zeitgedächtnis der Bienen biologische Bedentung Z VgI Physiol 22, 221-262

Masson C (1983) Rôle des médiateurs chimiques d'origine animale et végétale dans la pollinisation. CR Ve Symp Int Pollinisation, Versailles, 25-37

Mesquida J, Renard M (1978) Entomophilous pollination of male sterile strains of winter rapeseed (Brassica napus $L$ var oleifera) and a preliminary study of alternating devices. Proc IVth Int Symp Pollination, Maryland, USA, 1, 49-57

Mesquida J, Renard M, Pellan-Delourme R, Pelletier G, Morice J (1988 a) Influence des sécrétions nectarifères des lignées mâles-stériles pour la production de semences hybrides $F_{1}$ de colza. In : Variabilité génétique cytoplasmique et stérilité mâle cytoplasmique. Les Colloques de I'INRA, SainteSabine, France, 45, 269-280

Mesquida J, Marilleau R, Pham-Délègue MH, Renard M (1988 b) A study of rapeseed (Brassica napus $L$ var oleifera Metzger) flower nectar secretions. Apidologie 19, 307-318

Meyerhoff G (1958) Zum sammelverhalten der Bienen im Raps. Bienzeitung Liepzig 72, 164-165

Mitchener AV (1927) Influence of temperature on the honeyflow. Am Bee J 67, 120-121

Nunez JA (1973) Le comportement de butinage de l'abeille (Apis mellifera L). Étude quantitative du comportement. CR Congr Int Apiculture Apimondia, Buenos-Aires, 285-290

Oertel E (1946) Effect of temperature and relative humidity on sugar concentration of nectar. J Econ Entomol 39, 513-515

Ogura $H$ (1968) Studies of the new male sterility in Japanese radish, with special references to the utilization of this sterility towards the practical raising of hybrid seeds. Mem Fac Agric Kagoshima Univ 6, 39-78

Pelletier G, Primard C, Vedel F, Remy R, Rousselle P, Renard $M$ (1983) Intergeneric cytoplasmic hybridization in Cruciferae by protoplast fusion. Mol Gen Genet 191, 244-250

Percival MS (1961) Types of nectar in Angiosperms. New Phytol 60, 235-281

Pham-Délègue $\mathrm{MH}$, Fonta $\mathrm{C}$, Masson $\mathrm{C}$, Douault $\mathrm{P}$ (1985) Étude comparée du comportement de butinage d'insectes pollinisateurs (abeilles: Apis mellifica $L$ et bourdons : Bombus terrestris $L$ ) sur les lignées parentales d'hybrides de tournesol Helianthus annuus L Acta Oecol Oecol. Applic 6, 47-67

Pham-Délègue $M H$, Masson $C$, Etievant $P$, Azar $M$ (1986) Selective olfactory choices of the honeybee among sunflower aromas: a study by combined olfactory conditioning and chemical analysis. $J$ Chem Ecol 12, 781-793

Quemener B, Mercier C (1980) Dosage rapide des glucides éthanolo-solubles des graines de légumineuses par chromatographie liquide sous haute pression. Lebesm Wiss Technol 13, 7-12 
Radchenko TG (1964) The influence of pollination on the crop and the quality of seed of winter rape. Bradzhil Nitstro 1, 68-74 (en ukrainien)

Renard M, Mesquida J, Pellan-Delourme R, Pelletier G, Morice J (1988) Pollinisation des cybrides mâles stériles dans un système de culture mixte de colza. In : Variabilité génétique cytoplasmique et stérilité mâle cytoplasmique. Colloques INRA, SainteSabine, France, 45, 281-292

Robacker DC, Flottum PK, Sammataro D, Erickson EH (1983) Effects of climatic and edaphic factors on soybean flowers and on the subsequent attractiveness of the plants to honeybees. Field Crops Res 6 , 267-278

Severson DW, Erickson EH (1983) High performance liquid chromatography of carbohydrates in cucumber nectar J Apic Res 22, 158-162

Shuel RW (1955) Nectar secretion. Ann Bee J 95, 229-234

Thean JE, Fundersburk WC (1977) High pressure liquid chromatographic determination of sucrose in honey. J Assoc Off Anal Chem 60, 4, 838-841
Vear $F$, Pham-Délègue $M H$, Tourvieille de Labrouhe $D$, Marilleau R, Loublier $Y$, Le Métayer M, Douault P. Phillipon JP (1990) Genetical studies of nectar and pollen production in sunflower. Agronomie 10, 219-232

Vedel F, Chetrit P, Delourme R, Renard M, Pelletier G, Primard C (1987) Chloroplast and mitochondrial DNA variations between different CMS lines of rapeseed. CR VIle Congr Int Colza Poznan, 187-191

Waller GD, Carpenter EW, Ziehl OA (1972) Potassium in onion nectar and its probable effect on attractiveness of onion flowers to honeybees. $J A m$ Soc Hortic Sci 97, 535-539

Weast RC, Astle MJ, Beyer WH (1988) CRC Handbook of Chemistry and Physics, $68^{\circ}$ ed CRC Press D230-231-262

Williams IH (1980) Oil-seed rape and beekeeping, particularly in Britain. Bee World 4, 141-150

Wykes GR (1952) An investigation of the sugars present in the nectar of flowers of various species. New Phytol 51, 210-215 